\title{
Cost-effectiveness of introducing a rotavirus vaccine in developing countries: The case of Mexico
}

\author{
Atanacio Valencia-Mendoza*†1, Stefano M Bertozzi ${ }^{\dagger 2,3,4}$, Juan- \\ Pablo Gutierrez ${ }^{\dagger 5}$ and Robbin Itzler ${ }^{\dagger 6}$
}

\begin{abstract}
Address: ${ }^{1}$ Division of Health Economics, National Institute of Public Health, Av. Universidad No. 655, Col. Santa María Ahuacatitlán, Cuernavaca, Morelos, 62508, México, ${ }^{2}$ Center for Evaluation Research and Surveys, National Institute of Public Health, Av. Universidad No. 655, Col. Santa María Ahuacatitlán, Cuernavaca, Morelos, 62508, México, ${ }^{3}$ Haas School of Business, University of California, Berkeley, USA, ${ }^{4}$ Center for Economic Education and Research (CIDE), Mexico City, México, ${ }^{5}$ Division of Health Surveys, National Institute of Public Health, Av. Universidad No. 655, Col. Santa María Ahuacatitlán, Cuernavaca, Morelos, 62508, México and ${ }^{6}$ Merck \& Co., Inc., UG1C-60, P.O. Box 1000, North Wales PA 194541099, USA
\end{abstract}

Email: Atanacio Valencia-Mendoza* - avalencia@correo.insp.mx; Stefano M Bertozzi - sbertozzi@correo.insp.mx; JuanPablo Gutierrez - jpgutier@correo.insp.mx; Robbin Itzler - robbin_itzler@merck.com

* Corresponding author †Equal contributors

Published: 29 July 2008

BMC Infectious Diseases 2008, 8:103 doi:10.1186/1471-2334-8-103
Received: 3 December 2007

Accepted: 29 July 2008

This article is available from: http://www.biomedcentral.com//47/-2334/8//03

(c) 2008 Valencia-Mendoza et al; licensee BioMed Central Ltd.

This is an Open Access article distributed under the terms of the Creative Commons Attribution License (http://creativecommons.org/licenses/by/2.0), which permits unrestricted use, distribution, and reproduction in any medium, provided the original work is properly cited.

\begin{abstract}
Background: In developing countries rotavirus is the leading cause of severe diarrhoea and diarrhoeal deaths in children under 5 . Vaccination could greatly alleviate that burden, but in Mexico as in most lowand middle-income countries the decision to add rotavirus vaccine to the national immunisation program will depend heavily on its cost-effectiveness and affordability. The objective of this study was to assess the cost-effectiveness of including the pentavalent rotavirus vaccine in Mexico's national immunisation program.

Methods: A cost-effectiveness model was developed from the perspective of the health system, modelling the vaccination of a hypothetical birth cohort of 2 million children monitored from birth through 60 months of age. It compares the cost and disease burden of rotavirus in an unvaccinated cohort of children with one vaccinated as recommended at 2, 4 , and 6 months.

Results: Including the pentavalent vaccine in the national immunisation program could prevent 7I,464 medical visits (59\%), 5,040 hospital admissions (66\%), and 612 deaths from rotavirus gastroenteritis (70\%). At US $\$ 10$ per dose and a cost of administration of US $\$ 13.70$ per 3-dose regimen, vaccination would cost US\$122,058 per death prevented, US\$4,383 per discounted life-year saved, at a total net cost of US\$74.7 million dollars to the health care system. Key variables influencing the results were, in order of importance, case fatality, vaccine price, vaccine efficacy, serotype prevalence, and annual loss of efficacy. The results are also very sensitive to the discount rate assumed when calculated per life-year saved.

Conclusion: At prices below US $\$ 15$ per dose, the cost per life-year saved is estimated to be lower than one GNP per capita and hence highly cost effective by the WHO Commission on Macroeconomics and Health criteria. The cost-effectiveness estimates are highly dependent upon the mortality in the absence of the vaccine, which suggests that the vaccine is likely to be significantly more cost-effective among poorer populations and among those with less access to prompt medical care - such that poverty reduction programs would be expected to reduce the future cost-effectiveness of the vaccine.
\end{abstract}




\section{Background}

Worldwide, infection with rotavirus is the leading cause of severe diarrhoea in children under age 5 . Before age 5 , most children will have experienced an episode of rotavirus gastroenteritis. Rotavirus infection may be either asymptomatic or symptomatic. In the latter case, it is associated with acute gastroenteritis, beginning with an acute episode of watery diarrhoea, fever, and vomiting [1,2]. The diarrhoea lasts for an average of six days, although cases have been reported in which it has lasted up to 20 days [3]. Rotavirus infection is typically more severe than other common causes of childhood diarrhoea, and is more likely to be associated with dehydration, hospitalisation, and death [1,4-6]. Some estimates have shown that $20 \%-40 \%$ of all hospital admissions and $20 \%$ of deaths from diarrhoea are attributable to rotavirus in children under age 5 [4]. Every year, rotavirus causes approximately somewhere between 352,000 and 592,000 deaths, 2 million hospital admissions, 25 million medical visits, and 111 million cases of gastroenteritis requiring only home care, in children under 5 years old [4]. As a result, 1 out of 5 children with rotavirus gastroenteritis will visit a physician; 1 out of 65 will be admitted to a hospital; and approximately 1 out of 293 will die [4].

In temperate climates, rotavirus diarrhoea appears mostly in winter, whereas in tropical climates seasonal patterns of the disease are less evident [7] Incidence of rotavirus disease is similar among children in developed and developing countries. However, children in developing countries have higher mortality rates due to several factors, including less access to oral rehydration and a higher prevalence of malnutrition. An estimated 1,205 children die from rotavirus disease every day, and $82 \%$ of those deaths occur among children in low-income countries. In fact, children in low-income countries are nearly 3, 6 and 237 times as likely to die from rotavirus as children in low middle, upper middle and high income countries, respectively [4] Therefore, the potential benefits of a rotavirus vaccine in low-income countries and in the poorest parts of middleincome countries such as Mexico could be very considerable.

A new pentavalent rotavirus vaccine (PRV) for the prevention of rotavirus gastroenteritis has been developed (RotaTeq ${ }^{\odot}$, Merck). The vaccine contains five live bovinehuman reassortant serotypes (G1, G2, G3, G4, and $\mathrm{P} 1 \mathrm{~A}[8])$. The vaccine is given by mouth in a three-dose schedule, starting at 6 weeks of age, with one- to twomonth intervals between doses. The vaccine has been proven safe; and when the three-dose schedule is given, its efficacy against G1-G4 rotavirus gastroenteritis of any degree of severity is $74.0 \%$ (66.8 to $79.9 \% 95 \% \mathrm{CI}$ ), whereas efficacy against severe G1-G4 gastroenteritis is $98.0 \%$ ( 88.3 to $100 \% 95 \% \mathrm{CI}$ ). Taking this into consider- ation, this vaccine could prevent many of the deaths and hospitalisations resulting from severe episodes of rotavirus gastroenteritis.

Mexico, like many other low- and middle-income countries is currently facing decisions about whether and how to incorporate newly available, expensive vaccines into its national vaccination program. Just within the last two years the government initially decided to make the monovalent vaccine available for a subset of counties with high infant mortality rates and more recently decided to expand vaccination to the entire country.

In most low- and middle-income countries the decision to add a rotavirus vaccine to the national immunisation program will depend heavily on the cost-effectiveness and affordability of the vaccine. We present here a model developed for such settings and projections from the model using Mexican data.

\section{Methods}

\section{Target population and perspective}

A cost-effectiveness model was developed to inform the decision about whether to include universal vaccination with PRV in a national immunization program.

The cost-effectiveness analysis compares the cost of treatment for rotavirus diarrhoea in the absence of a national immunisation program with the cost of a national immunisation program plus the cost of treatment for those cases not averted by immunisation. Three doses of PRV would be given by mouth during the first 6 months of life. Three cost-effectiveness ratios are estimated: the incremental cost of the program divided by (1) the number of cases prevented, (2) the number of deaths prevented, and (3) the life-years gained. Cost-effectiveness is estimated from the perspective of the health care system, taking into account both the medical costs associated with rotavirus diarrhoea (as if all medical care costs were borne by the system) and the costs of the immunisation program. Productivity benefits and costs or savings that accrue to families (e.g. transport and childcare) other than medical care were not included.

In the application of the model to Mexico, an annual birth cohort of two million children is assumed. Given the seasonality of rotavirus disease, the birth cohort is divided into 12 monthly sub-cohorts to distribute the births throughout the year, assuming a constant number of births per month. Costs are presented in 2006 dollars. All future costs and health benefits are discounted at 3\% in the base case. Rotavirus-associated medical costs are estimated for the first 5 years of life using age-specific incidence by season for each event. 


\section{Decision analysis model}

The costs and benefits of implementing a national rotavirus immunisation program were compared with a probabilistic model using spreadsheet based software (@RISK 4.5.4; Pallisade, Newfield, NY) in EXCEL (Figure 1). The model was analyzed under baseline conditions in order to determine the costs of either option: immunizing at the coverage levels of diphtheria and tetanus toxoids and pertussis (DTP) vaccine, or the prevailing situation, with no vaccine. Key variables used in the model were varied using univariate and probabilistic sensitivity analysis (see Tables 1 through 4 ).

In this model the current practice with no vaccination is compared with a vaccination program in which pentavalent rotavirus vaccine is included in a national childhood immunisation program at current levels of coverage for DTP. The vaccination program option represents inclusion of PRV in a national immunisation program, for a birth cohort of two million children (followed from birth to 5 years of age) divided into 12 monthly sub-cohorts of the same length. With a vaccination program, some children may not be vaccinated, and because vaccine efficacy is less than $100 \%$ against rotavirus diarrhoea, some vaccinated children also will become ill (Figure 1).

Given the perspective adopted in this study, the model does not distinguish between children who do not become ill with rotavirus diarrhoea and those who become ill and do not use healthcare services (unless they die). In other words, the model does not consider the potential benefit associated with preventing cases of diarrhoea that are treated at home. In the diarrhoea arm of the model are those who experience a medical visit, hospitalisation, or death.

In this model the cohort is followed from birth to 5 years of age and is allowed to have different incidence of rotavirus medical visits, hospitalisations and deaths, according to the month of the year and the age, in months, of the children. The medical visits, hospitalisations and deaths averted each month by the program correspond to the reduction of incidence of each outcome due to serotypespecific vaccine efficacy. The advantage of this approach is that it enables the modelled benefits to reflect the lack of vaccine benefit in reducing incidence prior to the first dose, as well as different levels of protection after the first, second and third doses. It also enables estimation of how benefits can vary because of the seasonality of the incidence of the virus. Once data become available in Mexico on the real ages at which children receive the vaccine (as compared to the ideal 2, 4, and 6 month vaccination schedule modelled here) it will be possible to model the expected reduction in benefit associated with delays in vaccination.

The model inputs including disease burden, medical costs, rotavirus serotypes prevalence, discount rate, efficacy, coverage and price of the vaccine are shown in Tables 1 to 4 .

\section{Rotavirus disease: morbidity and mortality}

Because the model estimates morbidity and mortality for each monthly sub-cohort, it is possible to estimate the expected number of cases, nationally, for each event in each sub-cohort, by month and age, in order to consider seasonal disease pattern as well as the seasonality of vac-

Table I: Disease parameters

\begin{tabular}{|c|c|c|c|c|c|c|c|}
\hline & \multirow{2}{*}{$\begin{array}{r}\begin{array}{r}\text { Monthly morbidity from rotavirus } \\
\text { diarrhoea as a percentage of diarrhoea } \\
\text { by all causes, 2002' }\end{array} \\
\text { Under age I }\end{array}$} & \multicolumn{3}{|c|}{$\begin{array}{l}\text { Monthly morbidity from diarrhoea } \\
\text { (per 100,000), 2003² }\end{array}$} & \multicolumn{3}{|c|}{$\begin{array}{l}\text { Monthly mortality from diarrhoea } \\
\text { (per } 100,000), 2002^{3}\end{array}$} \\
\hline & & Under age I & Age I to 4 & Birth to age 5 & Under age I & Age I to 4 & Birth to age 5 \\
\hline Jan & 62.6 & 289.3 & 228.6 & 296.4 & 10.88 & 1.2 & 3.1 \\
\hline Feb & 66.95 & 228.6 & 226.4 & 228.6 & 7.11 & 0.7 & 2 \\
\hline Mar & 70.43 & 217.9 & 285.7 & 226.4 & 6.22 & 0.7 & 1.8 \\
\hline Apr & 63.47 & 296.4 & 325 & 285.7 & 4.66 & 0.5 & 1.3 \\
\hline May & 46.08 & 342.9 & 321.4 & 325 & 5.66 & 0.6 & 1.6 \\
\hline Jun & 26.08 & 328.6 & 457.1 & 321.4 & 4 & 0.4 & I.I \\
\hline Jul & 1.73 & 482.1 & 335.7 & 457.1 & 5.33 & 0.6 & 1.5 \\
\hline Aug & 0.43 & 357.1 & 375 & 335.7 & 5.44 & 0.6 & 1.6 \\
\hline Sep & 8.69 & 300 & 296.4 & 375 & 4.22 & 0.4 & I.I \\
\hline Oct & 6.08 & 314.3 & 200 & 296.4 & 3.55 & 0.4 & I \\
\hline Nov & 29.56 & 225 & 150 & 200 & 4.77 & 0.5 & 1.3 \\
\hline Dec & 63.47 & 164.2 & 100 & 100 & 8 & 0.8 & 2.2 \\
\hline
\end{tabular}

I/Source: InDRE (Institute for Epidemiologic Diagnosis and Reference)

2/Source: Health Ministry and SUIVE (Unified Information System for Epidemiological Surveillance)

3/Source: INEGI (National Institute National Institute of Statistics, Geography and Informatics) 
Table 2: Health services utilization for children under 5 with diarrhoea

\begin{tabular}{lccrr}
\hline & Hospital admissions & Outpatient visits & Total & \% requiring hospitalization \\
\hline$<$ I year & 6,390 & 37,449 & 43,839 & $14.58 \%$ \\
I year & 4,250 & 110,805 & 115,055 & $3.69 \%$ \\
2 years & 4,997 & 89,481 & 94,478 & $5.29 \%$ \\
3 years & 2,367 & 56,810 & 59,177 & $4.00 \%$ \\
4 years & 1,459 & 40,180 & 41,639 & $3.50 \%$ \\
\hline
\end{tabular}

Source: Instituto Mexicano del Seguro Social (IMSS), 2005

cination campaigns when estimating the benefits of the vaccine. In this way the model accounts for potential differences in health benefit when a child is vaccinated just before the beginning of the high-risk season or just after it finishes. This is relatively unimportant for countries that predominantly vaccinate children at routine well-baby visits, but potentially quite significant for countries that vaccinate with annual or semi-annual vaccination campaigns.

The model assumes that serotype specific effectiveness of PRV is constant within a country or region of interest. This implies that the proportionate reduction of hospitalisations and outpatient visits for any particular serotype is assumed to be the same everywhere. This is supported by a recent analysis of the Rotavirus Efficacy and Safety Trial (REST) by region [8]. However, there are large differences among countries in the proportion of illness due to different serotypes as well as differences in severity of illness given underlying differences in nutritional and health sta- tus as well an availability of prompt and effective care for children with diarrhoea. Thus, use of the model in different settings requires calibrating it to local serotype incidence, and age-specific disease incidence and severity. In Table 3 we present the prevalence of G1-G4 serotypes specific for Mexico used in the model.

In the case of Mexico, nationally representative data are available for diarrhoea incidence for 0 - 11 months of age and for $12-59$ months of age as well as the proportion of diarrhoea in the $0-11$ month group that is due to rotavirus. However, there is no further breakdown by age of the diarrhoea incidence for Mexico. The IMSS, the Mexican Social Security system that cares for more than 30 percent of the population, has data on incidence by month in the first year of life and by year till age 5 . Because the IMSS population is wealthier on average than the non-IMSS population, the overall incidence of severe disease is lower, but the relative distribution of disease among the months/years is probably similar to that in the general

Table 3: Parameters used in the model

\begin{tabular}{|c|c|c|c|}
\hline & Baseline scenario & Range for sensitivity analysis & Source \\
\hline \multicolumn{4}{|l|}{ Efficacy for the first year following vaccination (\%) } \\
\hline Medical visits & 87.7 & $(76.2-93.7)$ & Vesikari T, et al, 2007 \\
\hline Hospital admissions & 96.3 & $(91.1-98.5)$ & Vesikari T, et al, 2007 \\
\hline \multicolumn{4}{|l|}{ Efficacy for the second year following vaccination (\%) } \\
\hline Medical visits & 73.8 & $(11.5-95.7)$ & Vesikari T, et al, 2007 \\
\hline Hospital admissions & 86.9 & $(10.8-99.7)$ & Vesikari T, et al, 2007 \\
\hline Prevalence of GI-G4 serotypes (\%) & 90.0 & $(83-97)$ & Mota-Hernandez, et al, 2003 \\
\hline Cost per medical visit) 2006 dollars) & 35.85 & $(34.59-37.29)$ & Nafate, 2005 \\
\hline Cost per hospital day (2006 dollars) & 222.55 & $(215.13-229.97)$ & Nafate, 2005 \\
\hline Cost of the vaccine per dose (2006 dollars) & 10.00 & & \\
\hline Cost of administration for the whole schedule (2006 dollars) & 13.77 & & \\
\hline Discount rate & $3 \%$ & & \\
\hline \multicolumn{4}{|l|}{ Life expectancy in years at age: } \\
\hline 0 years & 70.38 & & $\ddagger$ \\
\hline I years & 71.95 & & $\ddagger$ \\
\hline 2 years & 71.74 & & $\ddagger$ \\
\hline 3 years & 70.84 & & $\ddagger$ \\
\hline 4 years & 69.91 & & $\ddagger$ \\
\hline
\end{tabular}

‡ CONAPO's own estimates. Mortality tables, 2002. Available at http://www.conapo.gob.mx/00cifras/proy/RM.xls 
Table 4: Variables, values and distributions for the probabilistic sensitivity analysis

\begin{tabular}{llll}
\hline Variable & Values & Distribution \\
\hline $\begin{array}{l}\text { Reduction in outpatient visits } \\
\text { Reduction in hospitalization }\end{array}$ & Median $0.8775^{\text {th }}$ percentile $0.76295^{\text {th }}$ percentile 0.937 & Beta \\
Efficacy between the first and the third dose & Median $0.9635^{\text {th }}$ percentile $0.91195^{\text {th }}$ percentile 0.985 & Beta & Beta \\
Annual efficacy decrease & Median $0.7265^{\text {th }}$ percentile $0.5745^{\text {th }}$ percentile 0.828 & Beta & Gama \\
Outpatient visit cost & Median $0.15^{\text {th }}$ percentile $0.0595^{\text {th }}$ percentile 0.18 & Exponential \\
Hospitalization day cost & Median $34.595^{\text {th }}$ percentile $34.595^{\text {th }}$ percentile 37.29 & \\
\hline
\end{tabular}

population. Thus, the IMSS data were used to distribute incident cases among the months of year 1 and amongst the years 1-4 as required for the model (see Table 2).

There is also no information about how the proportion of diarrhoea cases due to rotavirus changes with age in Mexico. For children less than 1 year of age we only had the overall proportion of diarrhoea due to rotavirus so we used the distribution from the Marie-Cardine et al study from France to impute the distribution across the 12 months [9]. This distribution is critical because it enables quantification of changes in benefit associated with delays in vaccination due, for example, to campaign vaccination as opposed to routine vaccination. Mexican data are not

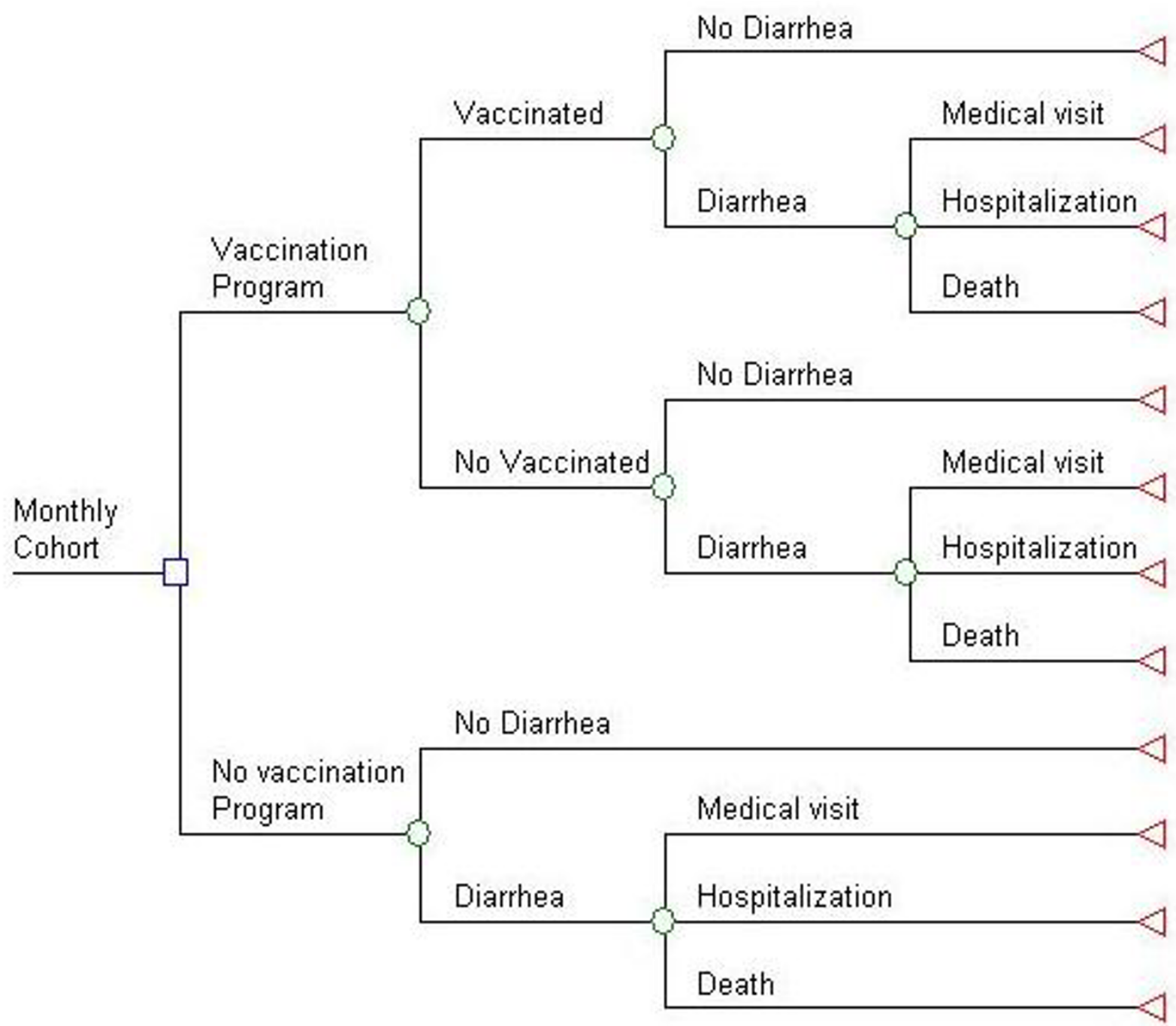

Figure I

Schematic representation of the model for rotavirus immunisation program in Mexico. 
available on the proportion of diarrhoea cases due to rotavirus among children one year or older, thus we used the proportions reported by Rivest et al from Canada, normalised to the overall proportion due to rotavirus in Mexico [10]. The normalisation was done comparing the overall proportion due to rotavirus $0-12$ mo in Canada with that in Mexico.

Table 1 shows the monthly morbidity from rotavirus diarrhoea in children under age 1 as a percentage of all cases of diarrhoea during 2002. The source for these estimates was a study carried out in secondary- and tertiary-care sentry hospitals in 15 large cities across Mexico, with data from 1996 through 2002 [11].

Data on the incidence and mortality associated with diarrhoea from all causes, as well as the fraction requiring medical visits or hospital admission, obtained either monthly or by age groups from various sources, are shown in Tables 1 and 2 .

In each monthly sub-cohort, representing infants born during 2006, the expected numbers of medical visits, hospital admissions and deaths attributable to rotavirus were estimated for every month of life up to the age of 5 (60 months). These estimates were based on the specific incidence by month and age group of events attributable to rotavirus (see Tables 1 and 2). Even though rotavirus diarrhoea is reported to be more severe on average than the other major causes of childhood diarrhoea, no Mexican data are available on how the proportion attributable to rotavirus differs among cases treated at home, cases treated in ambulatory settings, cases hospitalized, and deaths. The situation is further complicated by the fact that initial infection with rotavirus is likely to be more severe than subsequent infections, even by other serotypes [12]. To the extent that this is true, then the relative severity of rotavirus is likely to fall with age. In absence of data, we assumed that the proportion of diarrhoea cases due to rotavirus was constant across health care settings.

\section{Vaccine coverage levels, efficacy estimates, and prevalence of GI, G2, G3, and G4 serotypes in Mexico}

The vaccine coverage for PRV is anticipated to be the same as the one for the DTP vaccine, since both have a recommended schedule of administration at 2, 4, and 6 months of age. In 2004, the coverage of the full schedule of DTP vaccine was $94 \%$ in children under age 1 [13]. In the baseline scenario, we assume that the vaccine will be given during the first 6 months of life as recommended, with the three doses at 2, 4, and 6 months of age. Alternatively, to see how sensitive the results are to delays in vaccination, we estimated a scenario in which children are vaccinated at 6,8 and 10 months of age (maximum age permitted).
The efficacy estimates were taken from a clinical trial of PRV [8], containing five live human-bovine reassortant rotavirus strains (G1, G2, G3, G4, and P1A[8]). Efficacy estimates in this study show an $87.7 \%$ reduction in office visits from gastroenteritis caused by serotypes G1, G2, G3, and G4 (95\% CI, 76.2-93.7), and a 96.3\% reduction in hospital admissions from gastroenteritis caused by serotypes G1, G2, G3, and G4 (95\% CI, 91.1-98.5, Table 3). Efficacy against deaths from rotavirus gastroenteritis is assumed to be similar to efficacy in reducing hospitalisations. In the sensitivity analysis, the efficacy of the vaccine was varied within the range of its confidence interval in the clinical trial. These assumptions are conservative. The emergency room visits were combined with outpatient visits assuming the latter's efficacy, despite trial data suggesting a greater reduction in the more severe disease treated in emergency rooms because no information was available on the number of emergency room visits in Mexico. Similarly, one would expect deaths to be reduced more than hospitalisations.

The aforementioned efficacy estimates correspond to the first year starting 14 days after completion of the three dose regimen. Efficacy estimates are also reported for the second year, showing a slight decrease in protection against the disease (Table 3 ). To take into account the likelihood of decreasing protection conferred by PRV against rotavirus disease over time, and the lack of data on protection after the second year, the baseline scenario assumes an annual $10 \%$ efficacy reduction from year three to five. In the univariate sensitivity analysis, this percentage was varied from 0 to $30 \%$.

The vaccine clinical trials for PRV were powered to estimate the protection conferred by the full vaccination schedule and not the partial protection conferred after the first or second dose. However, the model must consider the effect of the vaccine before all three doses are administered. Two different efficacy estimates are available from the clinical trial: that from the intent-to-treat analysis, which includes all enrolled children, whether they received the full vaccine schedule or not, and the per-protocol analysis which only considers those who received the three doses per protocol. We considered the intent-totreat efficacy as the weighted average of the efficacy for full schedule and the efficacy for partial schedule and used that to estimate the effectiveness of a partial schedule. The estimation procedure was as follows:

In algebraic terms we have:

$$
\mathrm{E}_{\mathrm{PP}} *\left(\mathrm{P}_{\mathrm{PP}} / \mathrm{P}_{\mathrm{ITT}}\right)+\mathrm{X}^{*}\left(1-\mathrm{P}_{\mathrm{PP}} / \mathrm{P}_{\mathrm{ITT}}\right)=\mathrm{E}_{\mathrm{ITT}}
$$

Where: 
$\mathrm{E}_{\mathrm{PP}}=$ efficacy for the first year in the per-protocol study

$\mathrm{P}_{\mathrm{PP}}=$ subjects in the per protocol study

$\mathrm{P}_{\mathrm{ITT}}=$ subjects in the intent-to-treat study

$\mathrm{E}_{\mathrm{ITT}}=$ efficacy for the first year in the intent-to-treat study

$\mathrm{X}=$ efficacy between the first and the third dose, which is the unknown variable we want to estimate

Note that $\mathrm{P}_{\mathrm{PP}} / \mathrm{P}_{\mathrm{ITT}}$ is the weight corresponding to the subjects who received all three doses among all subjects enrolled, and that $\left(1-\mathrm{P}_{\mathrm{PP}} / \mathrm{P}_{\text {ITT }}\right)$ is the weight of those with an incomplete immunisation schedule. This approach doesn't provide information about how protection is different between the first and second dose from that between the second and the third. We have assumed constant effectiveness between the first and third dose.

Finally, the expression corresponding to the estimation is

$$
\mathrm{X}=\left(\mathrm{E}_{\mathrm{ITT}}-\mathrm{E}_{\mathrm{PP}} *\left(\mathrm{P}_{\mathrm{PP}} / \mathrm{P}_{\mathrm{ITT}}\right)\right) /\left(1-\mathrm{P}_{\mathrm{PP}} / \mathrm{P}_{\mathrm{ITT}}\right)
$$

The reported efficacy estimates relate to protection against disease caused by serotypes G1, G2, G3, and G4. To estimate the potential reduction in the number of target events (medical visits, hospitalisations, and deaths) achieved by the vaccine, the fraction of rotavirus gastroenteritis attributable to serotypes G1, G2, G3, and G4 will be taken into account; in Mexico, this figure is 90\% [14]. In the sensitivity analysis, this percentage was varied from 78 to $92 \%$, to account for the range reported in the literature.

\section{Cost estimates}

Medical costs include the costs of outpatient visits and hospital admissions, as well as the costs associated with immunisation. To reflect the heterogeneity of the Mexican Health System we considered the cost of illness estimated for the main health institutions. We used the costs estimated by Náfate-Martínez (2005) for the Ministry of Health, the IMSS (Mexican Social Security Institute), and private providers [15]. These system-specific estimates were averaged by weighting each by the proportion of people who receive treatment nationally from that system. The estimate for the IMSS was assumed to be representative of all social security institutions (it is by far the largest). Table 3 shows data on the estimated costs per outpatient visit and hospital day from gastroenteritis converted into US dollars at 10.89 pesos per dollar the exchange rate for September 8th, 2006. The average length of a hospital stay was estimated to be five days. In the probabilistic sensitivity analysis, cost per medical visit was varied using a gamma distribution and the cost per hospitalization using an exponential distribution (Table 4). For the univariate sensitivity analyses, the upper and lower bounds of the $95 \%$ confidence interval were used.

For our baseline scenario, the price for each dose of the vaccine was set at $\$ 10$ dollars. Furthermore, the cost for administering the full immunisation schedule, excluding the vaccine itself, was assumed to be 150 Mexican pesos and converted into US dollars at 10.89 pesos per dollar the exchange rate for September $8^{\text {th }}, 2006$.

Given the chosen perspective, we have only considered medical costs associated with either of the two alternatives (immunisation and no immunisation). A further analysis could encompass a societal perspective, including nonmedical costs for both alternatives, such as transportation costs and productivity losses.

\section{Life-years saved}

Once the number of deaths preventable by immunisation in our cohort was estimated, the life-years saved were also estimated, based on the mortality tables published by CONAPO (the Mexican National Population Council), from which age-specific life expectancies were obtained (Table 3) [16]. Life-years saved by the intervention were calculated as the sum of deaths preventable at each age, multiplied by life expectancy at that age, which were discounted at a three percent rate.

\section{Sensitivity analysis}

\section{Univariate sensitivity analysis}

Starting from the baseline scenario, univariate sensitivity analyses were carried out to examine the extent to which the uncertainty in the variables affects our estimates. Considering that a market price for the vaccine has not been set, a sensitivity analysis on this variable was also performed. This makes it possible to anticipate cost-effectiveness values for the program in a range of potential scenarios of price per dose.

Given that the rotavirus case-fatality rate changes over time (improvements in health care access, nutrition, poverty alleviation programs, etc.), we performed a separate analysis varying the actual case-fatality rate from $50 \%$ to $200 \%$ to see how sensitive the cost per life-year saved is throughout these different contexts.

\section{Probabilistic sensitivity analysis}

To explore the robustness of the model and to estimate a feasible variation range of the cost-effectiveness results we conducted probabilistic sensitivity analyses and ran a hundred thousand model iterations with random sampling from the distributions.

For each of the uncertain variables in the model we defined a range and distribution of variation for the prob- 
abilistic sensitivity analysis. Table 4 shows the variables used in the probabilistic sensitivity analysis, their values and assumed distributions.

Because the vaccine price has not yet been set by the manufacturer, the model was also estimated for across a range of possible prices from $\$ 5$ to $\$ 15$ dollars per dose.

\section{Results}

\section{Baseline scenario}

Our estimates suggest that if Mexican children were vaccinated as part of a national immunisation program, universal vaccination could prevent 71,464 medical visits (59\%), 5,040 hospital admissions (66\%), and 612 deaths from rotavirus gastroenteritis (70\%) (Table 5). Medical costs for rotavirus diarrhoea estimated for the cohort were 11.9 million dollars without the immunisation program. Assuming a $\$ 10$ dollar price per dose, the national immunization program would cost $\$ 82.3$ million dollars, and would save $\$ 7.6$ million dollars in medical costs. Thus, the national immunization program would have a net cost of $\$ 74.7$ million dollars, leading to a net cost per death prevented (or life saved) of $\$ 122,058$ dollars and a cost per discounted life-year saved of $\$ 4,283$ dollars (Table 5).

\section{Univariate sensitivity analysis}

The leading determinants of the cost-effectiveness results were, case fatality, vaccine price, vaccine efficacy, serotype prevalence, and annual loss of efficacy. When our model was evaluated at the lower limit of the efficacy parameters, the cost per life-year saved rose from $\$ 4,283$ to $\$ 6,598$ dollars $(+54 \%)$, whereas at the upper limit, our cost-effectiveness ratio falls to $\$ 3,969$ dollars $(-7.3 \%)$ per life-year saved (Figure 2). The asymmetry reflects the fact that in the base case the effectiveness is very high and has little room to improve.

On the other hand, our estimates are less sensitive to the medical costs of the disease. Varying the estimated costs

Table 5: Results in terms of health and costs, with and without the rotavirus vaccine program

\begin{tabular}{|c|c|c|c|c|}
\hline Variable & Without the program & With the program & $\begin{array}{r}\text { Preventable by the vaccine } \\
\left(5^{\text {th_-9 }} 5^{\text {th }} \text { percentile }\right)^{*}\end{array}$ & $\%$ reduction \\
\hline Medical visits & 122,134 & 50,670 & $\begin{array}{r}71,464 \\
(68,135-74,432)\end{array}$ & $59 \%$ \\
\hline Hospital admissions & 7,585 & 2,545 & $\begin{array}{r}5,040 \\
(4,851-5,186)\end{array}$ & $66 \%$ \\
\hline Deaths & 871 & 259 & $\begin{array}{r}612 \\
(593-628)\end{array}$ & $70 \%$ \\
\hline Life-years saved & & & $\begin{array}{r}43,411 \\
(42,092-44,570)\end{array}$ & \\
\hline Discounted Life-years saved & & & $\begin{array}{r}17,430 \\
(16,888-19,908)\end{array}$ & \\
\hline \multicolumn{5}{|l|}{ Medical costs (thousands of dollars) } \\
\hline For medical visits & $\$ 4,046.74$ & $\$ 1,671.90$ & $\begin{array}{r}\$ 2,374.84 \\
(2,134-2,638)\end{array}$ & $59 \%$ \\
\hline For hospital admissions & $\$ 7,905.79$ & $\$ 2,649.22$ & $\begin{array}{r}\$ 5,256.57 \\
(293-17,825)\end{array}$ & $66 \%$ \\
\hline Cost of the vaccine & & $\$ 56,400.00$ & $\begin{array}{r}-\$ 56,400.00 \\
(\mathrm{NA})\end{array}$ & \\
\hline Cost of administration of the vaccine & & $\$ 25,895.32$ & $\begin{array}{r}-\$ 25,895.32 \\
(\mathrm{NA})\end{array}$ & \\
\hline Total medical costs & $\$ 11,952.53$ & $\$ 86,616.44$ & $\begin{array}{r}-\$ 74,663.91 \\
(-79,624--62,049)\end{array}$ & \\
\hline Cost per case prevented (dollars) & $\begin{array}{r}\$ 968.23 \\
(796-1,054)\end{array}$ & & & \\
\hline $\begin{array}{l}\text { Cost per hospitalization prevented } \\
\text { (dollars) }\end{array}$ & $\begin{array}{r}\$ 14,814.88 \\
(12,277-16,045)\end{array}$ & & & \\
\hline Cost per death prevented (dollars) & $\begin{array}{r}\$ 122,058.40 \\
(101,113-131,226)\end{array}$ & & & \\
\hline Cost per life-year saved (dollars) & $\begin{array}{r}\$ 1,719.93 \\
(1,424-1,848)\end{array}$ & & & \\
\hline $\begin{array}{l}\text { Cost per life-year saved } \\
\text { (dollars, Discounted life-years) }\end{array}$ & $\begin{array}{r}\$ 4,283.75 \\
(3,548-4,606)\end{array}$ & & & \\
\hline
\end{tabular}

$* 5^{\text {th }}$ to $95^{\text {th }}$ percentile of the probabilistic estimates 


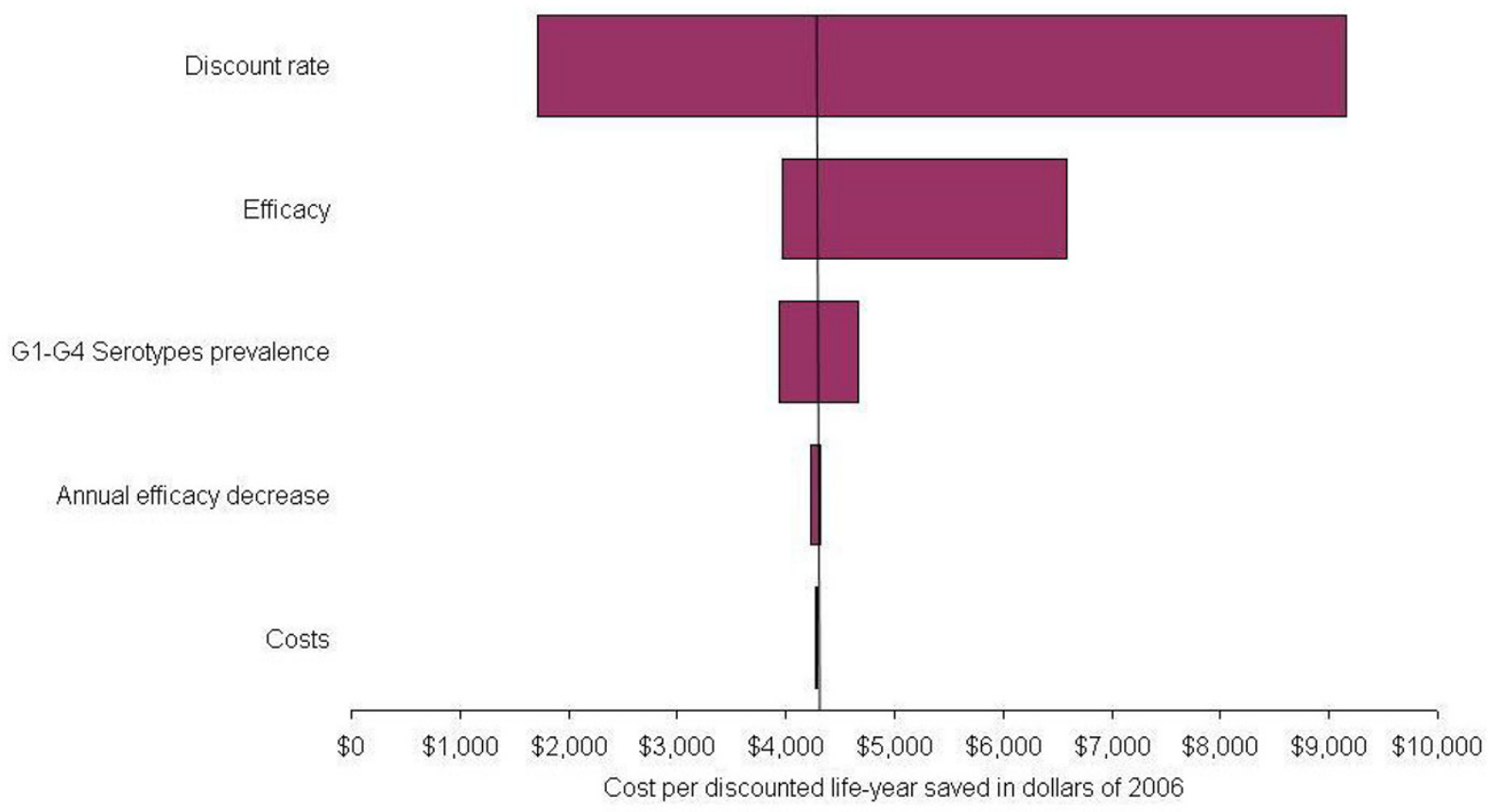

Figure 2

Univariate sensitivity analysis.

by the width of the confidence interval leads to a $-0.3 \%$ to $+0.4 \%$ variation in the cost per life-year saved (Figure 2 ). The results are very sensitive to the discount rate assumed when calculated per life-year saved because all costs are incurred in the first couple of years of life, but benefits continue for a lifetime. The model is not sensitive to discount rate when calculated per case of diarrhoea, hospitalisation or death averted because the costs and benefits occur concurrently.

When we calculated per life-year saved, our cost-effectiveness estimates are highly sensitive to variations in casefatality rate. Assuming a case-fatality rate 50\% lower than the actual rate, the model yields a cost per life-year gained of US\$8,566 (a 100\% increase), and assuming a casefatality-rate twice the actual rate, the cost per life-year gained is US\$2,142 (a 50\% decrease) (Figure 3).

When assuming a scenario in which children are vaccinated at 6,8 and 10 months of age, deaths averted fell to 501 (a 18\% decrease), while the cost per discounted lifeyear saved increased to US\$5,292 (a 19\% increase).

\section{Probabilistic sensitivity analysis}

For a price scenario of $\$ 10$ dollars per dose we ran a hundred thousand iterations using the parameter distributions defined in Table 4. The output for cost per discounted life-year saved is graphed in Figure 4. Ninety percent of the resulting cost-effectiveness estimates fell in the interval of $\$ 3,548$ to $\$ 4,606$ dollars per discounted life-year saved.

The results for the rest of the output variables are presented in brackets in Table 5 for the $5^{\text {th }}$ and $95^{\text {th }}$ percentile of the estimates. Ninety percent of the estimated averted deaths fell in the interval of 593 to 628 . The same interval for discounted life-years saved was 16,888 to 19,908 and for net cost it was 62 to 79.6 million dollars.

Figure 5 presents the results of the same sensitivity analysis conducted across the range of prices per dose. The shaded area bounded by the dotted lines is the area in which ninety percent of the estimates fall, for each price. Given that there is no correlation between vaccine price and the uncertainty of any of the other parameters, the size of the interval does not vary with changes in price. The horizontal line at $\$ 7,310$ represents the 2005 Mexican GNP per capita in US dollars, as point of reference.

\section{Discussion}

At prices below US \$15 per dose, using base-case parameter values, the cost per life-year saved is estimated to be lower than one GNP per capita. This is a very conservative estimate because it ignores the benefits associated with preventing non-fatal cases of rotavirus, with the exception of the savings in health care costs. Given the absence of 


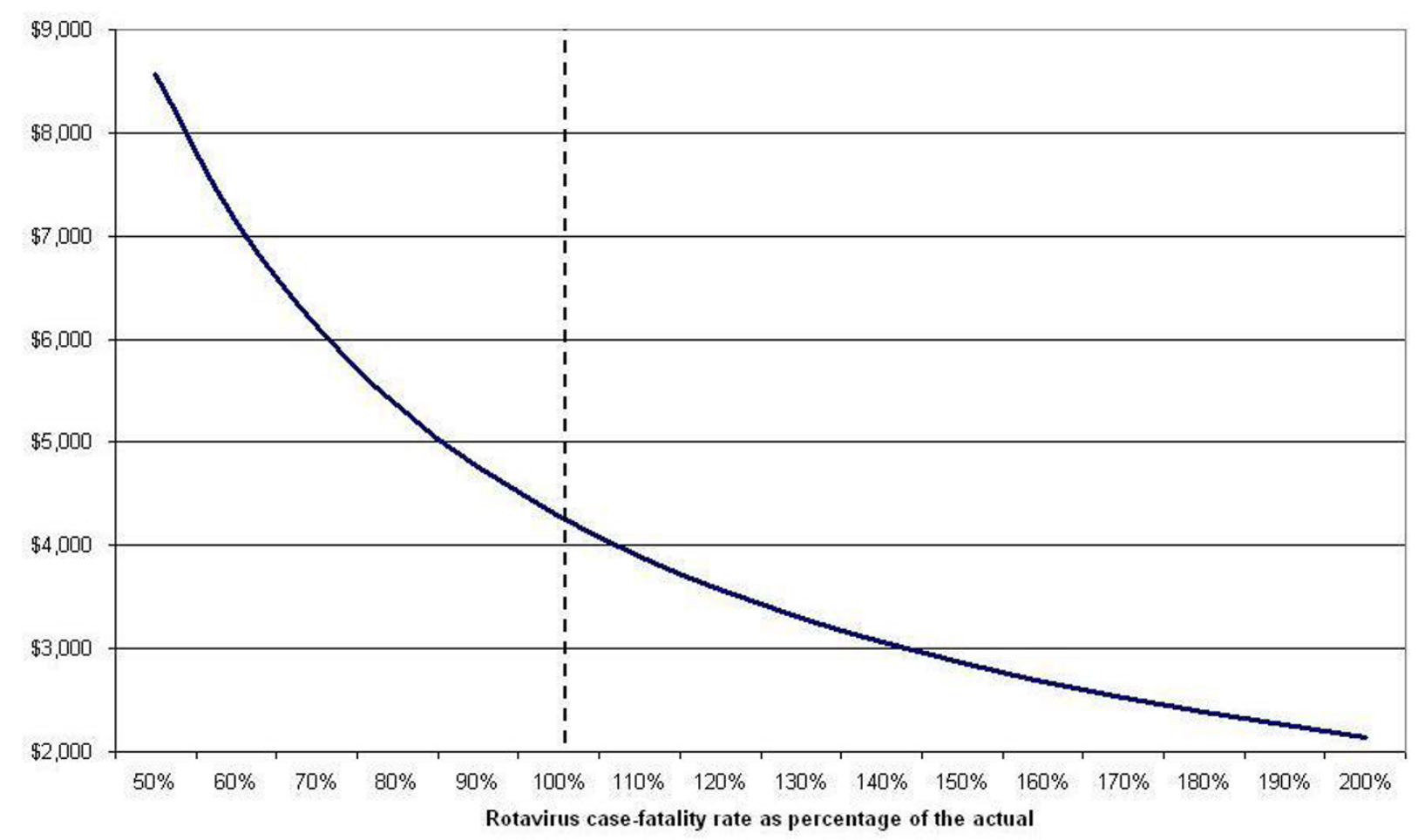

Figure 3

Relationship between rotavirus case-fatality rate and cost per discounted life-year saved (US dollars of 2006).

other strategies to prevent rotavirus infection and the fact that Mexico has already widely implemented a national public health program using oral rehydration therapy to reduce deaths from childhood diarrhoea, there was no obvious alternative intervention against which vaccination can be compared. However, the WHO Commission

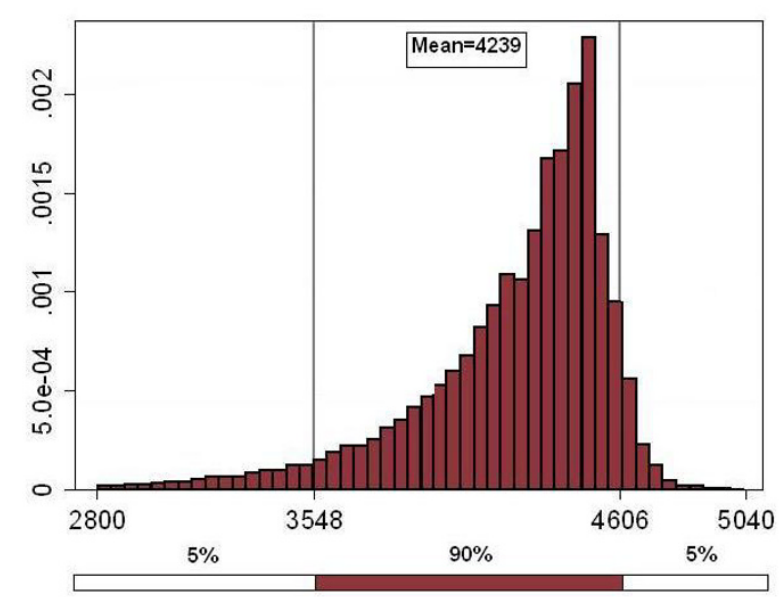

\section{Figure 4}

Cost per discounted life-year saved (US dollars of 2006). on Macroeconomics and Health suggested that interventions are highly cost-effective if the cost per life-year saved is lower than the per capita GDP; may be cost-effective if their cost is less than two to three times the per capita GDP; and are cost-ineffective if their cost is higher than three times the per capita GDP [17]. This suggests that a rotavirus immunisation program would be highly costeffective across a wide range of price-per-dose scenarios.

We estimated that delaying vaccination four months could reduce by $18 \%$ the number of deaths prevented with respect to vaccinating at the recommended ages. This result gives us some lights of the expected reduction in effectiveness for countries where most vaccination is conducted in campaigns where delays are caused by waiting till the next campaign.

The most common manner in which morbidity and mortality are simultaneously considered is via cost-utility analysis, using a measure of effectiveness such as quality adjusted life years (QALYs). However, in the case of rotavirus infection it would not be difficult to argue that the impact of the illness on parental leisure and productivity is a greater burden for households than the decrement in the quality of life of the infant. As a result, a willingness to pay study might be more useful for capturing the value of 


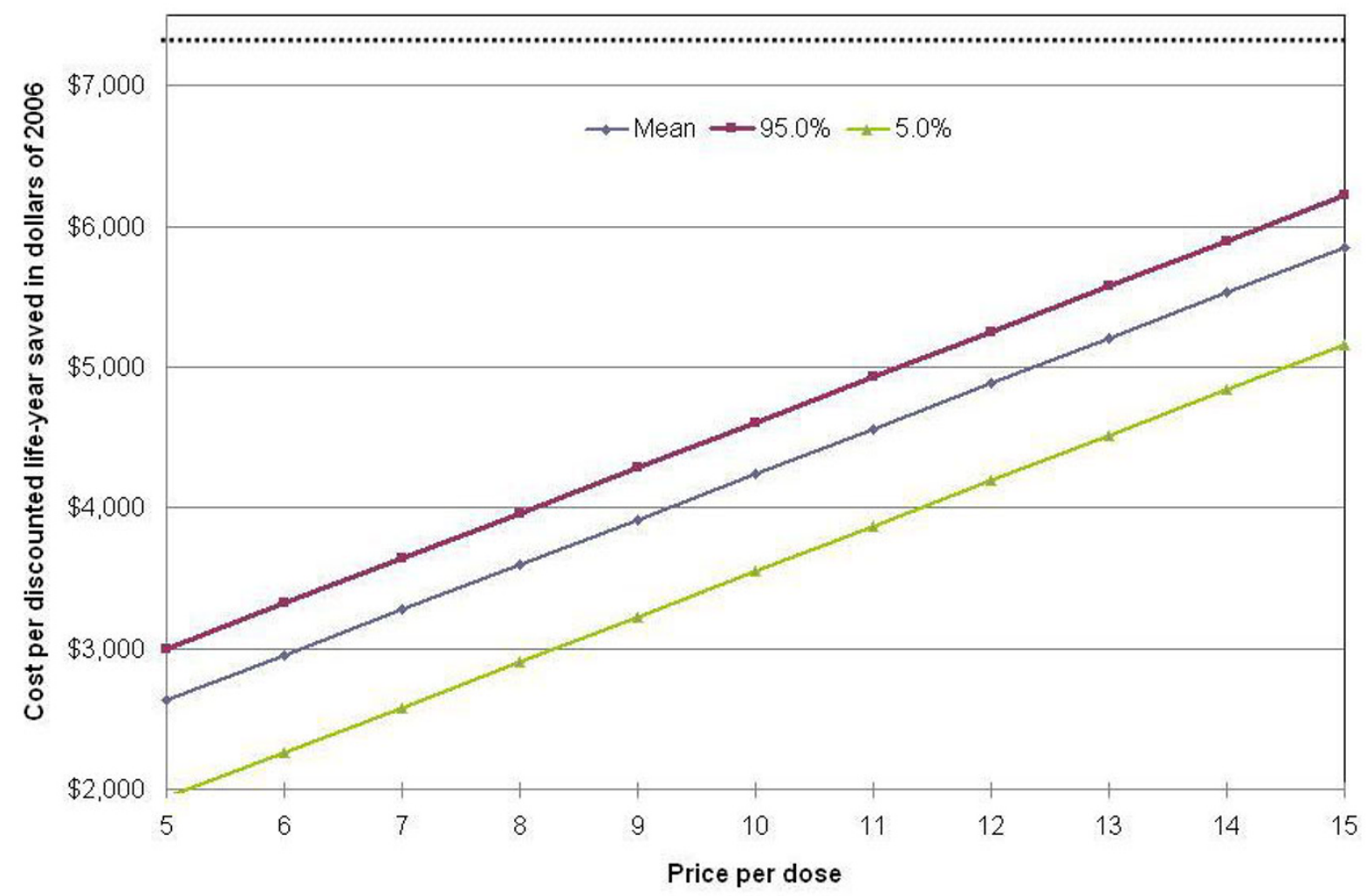

\section{Figure 5}

Probabilistic sensitivity analysis.

benefits of vaccination beyond mortality than a cost-utility analysis.

As with any vaccine, estimates of cost-effectiveness are highly dependent upon the expected morbidity and mortality in the absence of the vaccine. The estimates presented here reflect Mexican national averages in rotavirusassociated morbidity and mortality. While the incidence of infection with rotavirus is not strongly correlated with socioeconomic status, the likelihood that an infection results in severe dehydration requiring hospitalisation, or in death, is strongly negatively correlated with socioeconomic status. This suggests that the vaccine is likely to be significantly more cost effective among poorer populations and among those with less access to prompt medical care. It also suggests that poverty reduction programs, nutritional support programs and the introduction of health insurance programs for the poor, such as the ones recently introduced in Mexico would be expected to reduce the cost-effectiveness of the vaccine in the future.

As with any modelling exercise, the necessary simplification of a complex reality implies limitations that must be considered in the application of the results of the modeling exercise to public policy. In almost every case the sim- plifications reflected in this model would tend to underestimate the true cost-effectiveness of the vaccine. The exception is that the model does not consider possible vaccine side effects that might become apparent with large-scale implementation of the vaccine even though they were not observed in the clinical trial. Although none of the clinical trials or post-market surveillance to date suggest that this vaccine has significant vaccine-related mortality or morbidity, there always exists the possibility that a very low frequency side effect has not yet been detected - as occurred with RotaShield. However, given the high burden of Rotavirus disease in Mexico and other developing countries, even if this were to occur it is very unlikely to significantly alter the cost-effectiveness of the new vaccine.

As mentioned above, the model does not consider the impact of illness on parental productivity or the value to households of averted illness. The cost of illness is likely to be underestimated because it included outpatient and inpatient costs, but did not separately estimate costs of emergency care. In addition, the sample of facilities used for costing did not include any tertiary-care facility. 
The model assumes that children who do not die of rotavirus infection have average age-specific life expectancy. To the extent that children who die of rotavirus are those who are poorer and/or have less access to health services and/or have other health problems including malnutrition, then their life expectance may be shorter and the estimated benefits optimistic. In an upper middle income country such as Mexico, this bias is unlikely to be important, especially because at almost any discount rate the marginal difference in life expectancy is irrelevant. However in low income countries with high levels of competing infant mortality this is likely to be more important.

It is worth highlighting the fact that the estimates of cost of administration and cost of illness are highly uncertain, based on small-scale localised studies. Fortunately, they are not major determinants of the resulting cost-effectiveness estimates.

Finally, while such cost-effectiveness results are an essential input into the decision-making process, they are by no means sufficient. For example, even though this model considers differences in rotavirus mortality in populations of different socio-economic status, it does not weigh the distributional or equity implications of implementing rotavirus vaccination. Because the intervention disproportionally benefits the poorest segments of the population, a government that was seeking to reduce overall inequality in the population might give it higher priority than the simple CE results would suggest. Similarly, political and operational considerations are likely to be very important.

\section{Conclusion}

At prices below US $\$ 15$ per dose, the cost per life-year saved is estimated to be lower than one GNP per capita and hence highly cost effective according to the suggested criteria of the WHO Commission on Macroeconomics and Health. Cost-effectiveness estimates are highly dependent upon the case fatality in the absence of the vaccine, which suggests that the vaccine is likely to be significantly more cost effective among poorer populations and among those with less access to prompt medical care suggesting also that poverty reduction programs would be expected to reduce the cost-effectiveness of the vaccine in the future. In other middle-income countries with similar epidemiology (including case-fatality) the results are likely to be similar. However, it is more difficult to extrapolate these conclusions to low income countries. On the one hand, the case fatality is likely to be significantly higher and the cost of administering the vaccine lower because of lower labour costs (although in some countries this will be outweighed by higher transport and logistics costs), both of which would improve the vaccine's costeffectiveness. Whether this improvement in cost-effective- ness is sufficient to compensate for the much lower ability to pay will depend on the situation in a particular country.

As with any economic evaluation, caution should be exercised in extrapolating the results to other settings. In this case, the results of the modelling exercise are likely to be most sensitive to differences in rotavirus case fatality rates (see Figure 3) because these differ so much among developing countries. Vaccine efficacy is likely to vary relatively little if administered as recommended, but poorer countries are less likely to be able to administer the vaccine at 2, 4 and 6 months, significantly reducing its effectiveness in preventing infant deaths. Other potentially significant factors include differences in cost of adding the vaccine to the current regimen and differences in viral subtypes.

Finally, it is worth highlighting that while rotavirus vaccination represents an opportunity to reduce the burden of diarrhoeal disease in a cost-effective way in the short term, medium to long term solutions such as interventions to enhance health care seeking, education for oral rehydration therapy, health care infrastructure and nutrition improvements, among others, could be highly cost-effective and should be promote by developing countries.

\section{Competing interests}

This study was funded by Merck \& Co., Inc., which manufactures the vaccine under the brand name RotaTeq. Robbin Itzler is a full-time employee of Merck \& Co., Inc and she also owns stock and holds stock options in the company. Atanacio Valencia-Mendoza, Stefano M. Bertozzi and Juan-Pablo Gutierrez have no other competing interests other than sponsorship of this study by Merck \& Co., Inc.

\section{Authors' contributions}

$\mathrm{AV}-\mathrm{M}$ conceived the study, conducted the analysis of the data, interpreted the results and led the writing of the manuscript. SMB assisted with the study design, interpretation of the results and the writing of the manuscript. J-PG assisted with the study design and with the data analysis. RI assisted with the study design and with the interpretation of the results. AV-M and SMB had full access to all the data in the study and take full responsibility for the integrity of the data and the accuracy of the data analysis. All authors read and approved the final manuscript

\section{References}

I. Rodriguez WJ, Kim HW, Arrobio JO, Brandt CD, Chanock RM, Kapikian AZ, Wyatt RG, Parrott RH: Clinical features of acute gastroenteritis associated with human reovirus-like agent in infants and young children. J Pediatr 1977, 9 I(2): I88-93.

2. Sabbaj $L$, De Petre EE, Gómez JA, Sordo ME: Rotavirus en la diarrea aguda. Arch Argent Pediatr 200I, 99(6):486-90.

3. Uhnoo I, Svensson L: Clinical and epidemiological features of acute infantile gastroenteritis associated with human rotavirus subgroups I and 2. J Clin Microbiol I986, 23(3):55 I-5. 
4. Parashar UD, Hummelman EG, Bresee JS, Miller MA, Glass RI: Global illness and deaths caused by rotavirus disease in children. Emerg Infect Dis 2003, 9(5):565-72.

5. Blanck RE, Greenberg HB, Kapikian AZ, Brown KH, Becker S: Acquisition of serum antibody to Norwalk Virus and rotavirus and relation to diarrhea in a longitudinal study of young children in rural Bangladesh. J Infect Dis 1982, I 45(4):483-9.

6. Mota-Hernández F, Gutiérrez-Camacho C, Villa-Contreras S, CalvaMercado ], Arias CF, Padilla-Noriega L, Guiscafré-Gallardo H: [Prognosis of rotavirus diarrhea]. Salud Publica Mex 200 I, 43(6):524-8.

7. Cook SM, Glass RI, LeBaron CW, Ho MS: Global seasonality of rotavirus infection. Bull World Health Organ 1990, 68(2): 171-7.

8. Vesikari T, Itzler R, Matson DO, Santosham M, Christie CD, Coia M, Cook JR, Koch G, Heaton P: Efficacy of a pentavalent rotavirus vaccine in reducing rotavirus-associated health care utilization across three regions (I I countries). Int J Infect Dis 2007, I I(Suppl 2):S29-35.

9. Marie-Cardine A, Gourlain K, Mouterde O, Castignolles N, Hellot MF, Mallet E, Buffet-Janvresse C: Epidemiology of Acute Viral Gastroenteritis in Children Hospitalized in Rouen, France. Clin Infect Dis 2002, 34(9): I I 70-8.

10. Rivest P, Proulx M, Lonergan G, Lebel MH, Bédard L: Hospitalisations for gastroenteritis: the role of rotavirus. Vaccine 22( I 5- 16):2013-7. 2004 May 7

II. Velázquez FR, Garcia-Lozano H, Rodriguez E, Cervantes Y, Gómez A, Melo M, Anaya L, Ovalle JC, Torres J, Diaz De Jesus B, Alvarez-Lucas C, Breuer T, Muñoz O, Kuri P: Diarrhea morbidity and mortality in Mexican children: impact of rotavirus disease. Pediatr Infect Dis J 2004, 23( 10 Suppl):SI49-55.

12. Velázquez FR, Matson DO, Calva JJ, Guerrero L, Morrow AL, CarterCampbell S, Glass RI, Estes MK, Pickering LK, Ruiz-Palacios GM: Rotavirus infections in infants as protection against subsequent infections. N Engl J Med 335(I4): I022-8. 1996 Oct 3

13. Secretaría de Salud: Salud México 2004: Información para la rendición de cuentas. Mexico 2005 [http://www.salud.gob.mx/unidades/evaluacion/ saludmex2004/sm2004.pdf]. ISBN 970-72I-267-5

14. Mota-Hernández F, Calva J], Gutiérrez-Camacho C, Villa-Contreras S, Arias CF, Padilla-Noriega L, Guiscafré-Gallardo H, de Lourdes Guerrero M, López S, Muñoz O, Contreras JF, Cedillo R, Herrera I, Puerto $\mathrm{FI}$ : Rotavirus diarrhea severity is related to the VP4 type in Mexican children. J Clin Microbiol 2003, 4 I (7):3 I 58-62.

15. Nafate A: Determinación de costos por caso de diarrea en menores de cinco años atendidos en tres subsistemas de atención a la salud en Morelos, México. In MSc thesis Escuela de Salud Pública de México; 2006.

16. República Mexicana: Probabilidades de fallecer por sexo y edad, 2005-2050 [http://www.conapo.gob.mx/00cifras/proy/ RM.xis]

17. Informe sobre desarrollo humano para México 2005 del Programa de las Naciones Unidas para el Desarrollo [http:// saul.nueve.com.mx/informes/index.html]

\section{Pre-publication history}

The pre-publication history for this paper can be accessed here:

http://www.biomedcentral.com/1471-2334/8/103/pre pub http.//www.biomedcentral.com/1471-2334/8/103 